\title{
Calculation of the parameter "deflection" in a new theory of the Earth's magnetic field
}

\author{
A. de Paor and E. Burke \\ Department of Electronic and Electrical Engineering, National University of Ireland, Dublin, Belfield, Dublin 4, Ireland
}

Received: 13 December 2001 - Revised: 30 January 2002 - Accepted: 7 February 2002

\begin{abstract}
In a recent paper on the theory of the Earth's magnetic field and key features of Sunspot activity (de Paor, 2001), a central role in the calculation of secular variations of the geomagnetic field was played by a newly-introduced parameter called the deflection (abbreviated def). In this note, the significance of def is elucidated and the method used to calculate it is explained.
\end{abstract}

\section{Introduction}

It has long been recognised that there is an element of mystery associated with the phenomenon of time-varying declination of the Earth's magnetic field. This may perhaps be highlighted by considering that if we pass a great circle through London and the Earth's magnetic poles at present, a compass needle at London lying in that circle would point at an angle of $24.05^{\circ}$ to the west. Yet, this is nowhere near London's current value of magnetic declination: that is about $1^{\circ}$ to the west. The value of declination has swung from about $11.5^{\circ} \mathrm{E}$ in 1576 , right through to $24^{\circ} \mathrm{W}$ in about 1800 , and has been returning towards the east ever since. All that variation has occurred, according to Malin and Bullard (1981), without much change in the positions of the Earth's magnetic poles. They surmised that this large change in declination might be associated with eddies in the fluid core. A totally different viewpoint was recently advanced by de Paor (2001). This is that there are actually two orthogonal currents flowing within the Earth, with energy exchange between them provided by the Hall Effect, shown to function as a perfect orthogonal axis energy transfer mechanism. The socalled annulus current, $i_{a}$, encircles the solid core and gives rise to the main dipolar field of the Earth. Its plane is taken to be perpendicular to the Earth's magnetic axis. It, acting alone, would give a declination of $-24.05^{\circ}$ (i.e. $24.05^{\circ}$ to the west) at London. The other current, the so-called dynamo current, $i_{d}$, emerges from the solid core, cuts across the an-

Correspondence to: A. de Paor (annraoi.depaor@ucd.ie) nulus current at right angles, penetrates the mantle, splits into two halves, one going north and one south, and returns to the solid core under the magnetic poles. Ampere's Circuital Theorem (de Paor, 2001) leads to the conclusion that this dynamo current would have no external magnetic field if the magnetic axis were coincident with the geographical axis, for then its symmetry would mean that the external field of each sheet flowing in the mantle would be exactly counterbalanced by the field of the current returning to the solid core along the magnetic axis.

However, as has been verified by de Paor in yet unpublished experiments on a physical model (an aluminium sphere with a replica of the dynamo current flowing within it) and supported by analogical mathematical reasoning, the asymmetry produced by the offset of the magnetic axis from the geographical axis is sufficient to produce an external field transverse to the dipolar field. The magnetic axis may be derived from that the geographical axis by translating the latter about $10^{3} \mathrm{~km}$ out of the plane of the $120^{\circ} \mathrm{E}-60^{\circ} \mathrm{W}$ circle of longitude, towards the Pacific Ocean, and rotating it clockwise through $11.5^{\circ}$, around the centre of the circle being viewed from the Pacific. In de Paor (2001), the transverse field is taken to be a location-specific hysteretic function of the dynamo current. Hysteresis is thought to be due to the presence of magnetic rocks in the Earth's crust and upper mantle, for the transverse magnetic flux lines pass through these rocks for a very significant part of their length.

The concept of deflection plays a central role in calculating declination at any location: indeed declination simply cannot be calculated without a knowledge of deflection. In an attempt to explain this, Fig. 1 indicates geomagnetic conditions at any chosen point on the Earth's surface. The $Y$ axis lies in the plane of $i_{a}$ (perpendicular to the axis through the magnetic poles) and $i_{d}$ magnetises along it. The annulus current $i_{a}$ magnetises along the $X$ axis. The $Z$ axis points directly down into the ground. The angle between the $X$ axis and geographical north is the angle of deflection, def. We count it positive if $X$ points west of geographic north. As mentioned above, def currently has the value $24.05^{\circ}$ at Lon- 


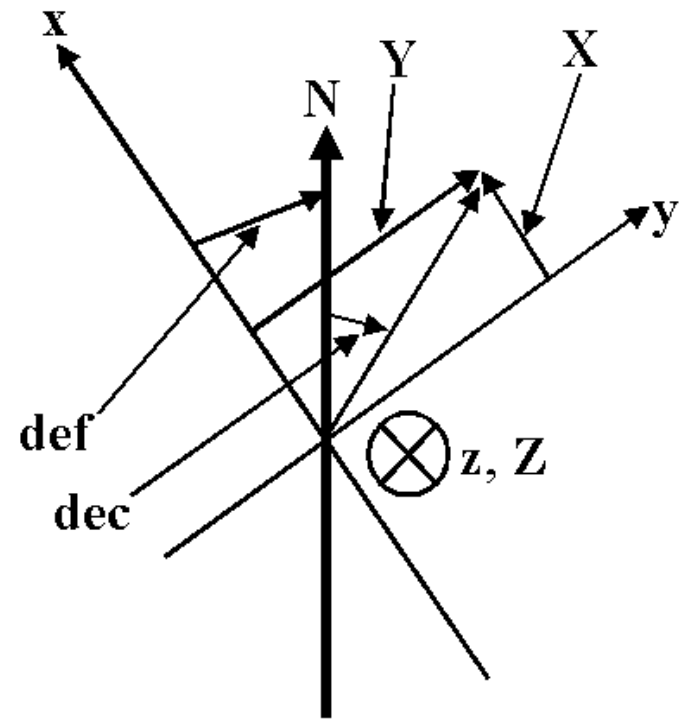

Fig. 1. Geometry to define def and calculate dec.

don (Greenwich) and in the view of Malin and Bullard (1981) this has changed little since the late 16th century. The transverse magnetising effect of the $i_{d}$ means that the horizontal component of the geomagnetic field vector, denoted by the vector $(\boldsymbol{X}, \boldsymbol{Y})$ on Fig. 1, points a net angle of $d e f+d e c$ to the east of the $X$ axis.

From Fig. 1, the equation governing dec is

$\operatorname{dec}=\tan ^{-1}(Y / X)-$ def.

In de Paor's theory, $X$ is proportional to $i_{a}$, while $Y$ depends hysteretically on $i_{d}$. Location-specific hysteresis is currently described by empirical functions and it remains a challenge, perhaps intractable, to find some theoretically justifiable expressions. When the fundamental significance of def was first appreciated by de Paor, it could not be calculated, and was measured directly from a globe for each location studied. The present authors have now co-operated to work out the theory of def. It turns out to be a reasonably straightforward but definitely non-trivial exercise in geometry on a sphere. The derivation is offered below in the hope of allowing de Paor's (2001) method of calculating declination to be extended to sites other than those reported on already.

\section{Calculation of the deflection}

Since the notation $(X, Y, Z)$ is used in a different context in Fig. 1, we shall use a right-handed orthogonal coordinate system $(q, r, s)$ in the development to follow. Lying in the plane of the paper on Fig. 2 is a great circle on the Earth's surface, on which lie $P$, the point whose geomagnetic field is under investigation, $N_{g}$, the geographic North Pole and $S_{g}$, the geographic South Pole. The great circle is in the $(q, r)$ plane, with the $s$ axis perpendicular to and directed out that. The latitude of $P$ is $\alpha$, measured positive above the $(q, s)$ plane. Normalising the radius of the Earth to unity, the coordinates

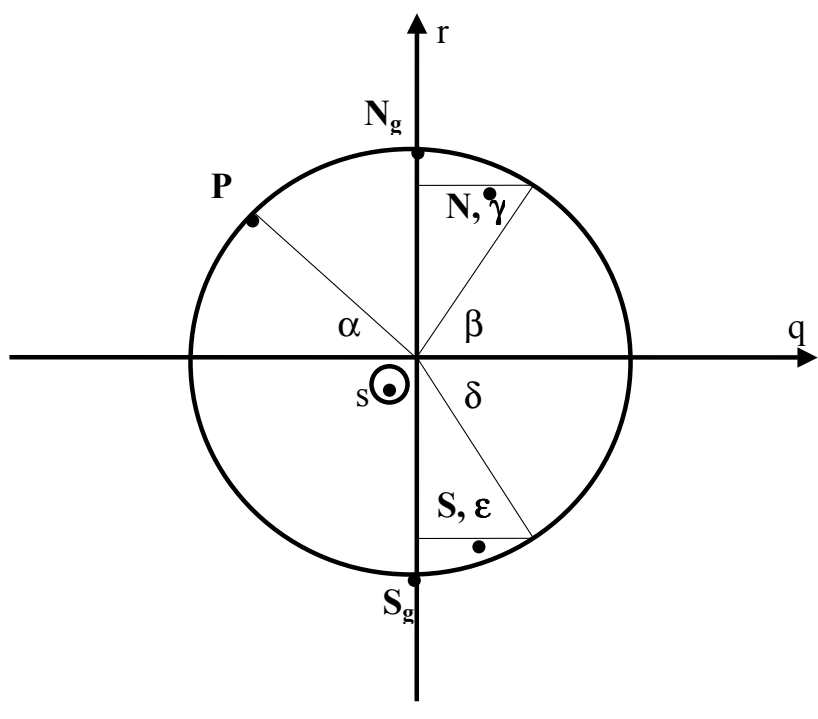

Fig. 2. Geometry for calculation of def.

of the three points mentioned are

$$
\begin{aligned}
P: & (-\cos \alpha, \sin \alpha, 0)=\left(q_{1}, r_{1}, s_{1}\right) \\
& N_{g}:(0,1,0) \\
& S_{g}:(0,-1,0) .
\end{aligned}
$$

We now consider the coordinates of the Magnetic North Pole, $N$. This is assumed to have latitude $\beta$, and to be rotated a net anticlockwise angle $\gamma$ from $P$, as viewed down along the $r$ axis. Thus, the coordinates of $N$ are

$N:(-\cos \beta \cdot \cos \gamma, \sin \beta, \cos \beta \cdot \sin \gamma)=\left(q_{2}, r_{2}, s_{2}\right)$.

The South Magnetic Pole, $S$, has latitude $\delta$, measured positive below the $(q, s)$ plane, and is rotated through a net anticlockwise angle $\epsilon$ from $P$, looking down along the $r$ axis. Thus, the coordinates of $S$ are

$S:(-\cos \delta \cdot \cos \epsilon,-\sin \delta, \cos \delta \cdot \sin \epsilon)=\left(q_{3}, r_{3}, s_{3}\right)$.

We now have three non-collinear points of interest, $P, N$ and $S$. The equation of the plane through them may be written (Rektorys, 1969)

$a . q+b . r+c . s=d$,

where

$$
\begin{aligned}
a= & \left(r_{1} s_{2}-r_{2} s_{1}\right)+\left(r_{2} s_{3}-r_{3} s_{2}\right)+\left(r_{3} s 1-r_{1} s_{3}\right) \\
& b=\left(s_{1} q_{2}-s_{2} q_{1}\right)+\left(s_{2} q_{3}-s_{3} q_{2}\right)+\left(s_{3} q_{1}-s_{1} q_{3}\right) \\
& c=\left(q_{1} r_{2}-q_{2} r_{1}\right)+\left(q_{2} r_{3}-q_{3} r_{2}\right)+\left(q_{3} r_{1}-q_{1} r_{3}\right) .
\end{aligned}
$$

The parameter $d$ is not needed here.

The vector $(a, b, c)$ is normal to the plane through $P, N$ and $S$. We multiply it by sign $(c)$ so that it is directed up out of the plane of the paper in Fig. 2. We then normalise it to get the unit upward normal,

$$
\begin{aligned}
\boldsymbol{n}= & (\operatorname{arsign}(c), \text { b.sign }(c), \operatorname{csign}(c)) / \\
& \left(a^{2}+b^{2}+c^{2}\right)^{1 / 2} .
\end{aligned}
$$


The unit upward normal to the $(q, r)$ plane is the vector

$\boldsymbol{m}=(0,0,1)$.

Since $\boldsymbol{n}$ is the upward unit normal to the plane containing $P, N$ and $S$, while $\boldsymbol{m}$ is the upward unit normal to the plane containing $P, N_{g}$ and $S_{g}$, it follows at once that

$\boldsymbol{m} \bullet \boldsymbol{n}=\cos (\operatorname{def})=c \cdot \operatorname{sign}(c) /\left(a^{2}+b^{2}+c^{2}\right)^{1 / 2}$,

where $\boldsymbol{m} \bullet \boldsymbol{n}$ is the scalar product of $\boldsymbol{m}$ and $\boldsymbol{n}$.

Equation (8) gives a positive or zero value of $\cos (\operatorname{def})$. The actual sign of def is determined by the sign of b.sign $(c)$, the $r$-coordinate of $\boldsymbol{n}$. If this is positive, def is directed to the west and and vice versa. Thus, our final expression for $d e f$ follows:

$\operatorname{def}=\operatorname{sign}(b) \cdot \operatorname{sign}(c) \cdot \arccos \boldsymbol{m} \bullet \boldsymbol{n}$.

\section{Examples:}

1. London (Greenwich), latitude $51.483^{\circ}$, longitude $0^{\circ} \Rightarrow$ $\operatorname{def}=24.05^{\circ}$,

2. Toolangi (Australia), latitude $-37.533^{\circ}$, longitude $145.467^{\circ} \Rightarrow \operatorname{def}=-9.4744^{\circ}$,

3. Cape of Good Hope, latitude $-33.933^{\circ}$, longitude $18.483^{\circ} \Rightarrow \operatorname{def}=14.617^{\circ}$

4. Hurbanovo (Slovakia), latitude 47.867, longitude $18.183 \Rightarrow$ def $=20.023^{\circ}$,

5. Palermo, latitude $38.12^{\circ}$, longitude $13.35^{\circ} \Rightarrow$ def $=$ $18.075^{\circ}$
6. Florence, latitude $43.78^{\circ}$, longitude $11.25^{\circ} \Rightarrow \operatorname{def}=$ $19.747^{\circ}$

7. Parc Saint-Maur (Paris), latitude $48.817^{\circ}$, longitude $2.5^{\circ} \Rightarrow \operatorname{def}=22.533^{\circ}$.

These are based on taking the North Magnetic Pole as being at latitude $73.5^{\circ}$, longitude $-96^{\circ}$, and the South Magnetic Pole at latitude $72.417^{\circ} \mathrm{S}$ (entered as positive in Eq. 3), longitude $155.267^{\circ}$.

\section{Discussion}

This note has been prepared to elucidate the role of def and to fill in the theory underlying Eq. (62) in de Paor (2001). It is planned to complete the picture in a further paper, justifying on physical and mathematical grounds the underlying assumption of that equation, that $i_{d}$ does in fact result in a transverse magnetisation, on account of the offset of the Earth's magnetic from its geographical axis.

\section{References}

de Paor, A.: A theory of the Earth's magnetic field and of sunspots, based on a self-excited dynamo incorporating the Hall effect, Nonl. Proc. Geophys., 8, 265-279, 2001.

Malin, S. R. C. and Bullard, E.: The direction of the Earth's magnetic field at London, Phil. Trans. Roy. Soc., A299, 357-423, 1981.

Rektorys, K.: Survey of applicable mathematics, Butterworth, London, (Translated from the Czech), 1969.

Shockley, W.: Electrons and holes in semiconductors, Van Nostrand, Princeton, NJ, 1950. 